\title{
Quantitative Assessment of Deformation-Induced Damage in a Semisolid Aluminum Alloy via X-ray Microtomography
}

\begin{abstract}
A.B. PHILLION, P.D. LEE, E. MAIRE, and S.L. COCKCROFT
Semisolid tensile testing combined with X-ray microtomography (XMT) was used to characterize the development of internal damage as a function of strain in an aluminum-magnesium alloy, AA5182. Novel techniques were developed to allow the quantification of both the size evolution and orientation of the damage to determine mechanisms controlling the early stage growth and localization. During the initial stages of semisolid deformation, it was observed that strain was accommodated by both the growth of as-cast porosity and the detection of new damage-based voids. As the volume fraction of damage increases, the growth of voids occurs in an orientation perpendicular to the loading direction, both through expansion within the grain boundary liquid and void coalescence. The damage then localizes, causing failure.
\end{abstract}

DOI: $10.1007 / \mathrm{s} 11661-008-9584-4$

(C) The Minerals, Metals \& Materials Society and ASM International 2008

\section{INTRODUCTION AND BACKGROUND}

THE control of defects during the processing of metals is critical to the production of high quality products. In the context of strain-induced defects, damage initiates as vacancies that coalesce and subsequently grow, resulting in macroscopic flaws. One example of this process is the formation of internal voids when semisolid material is strained. In direct chill casting of aluminum ingots and billets, the thermal stresses are sufficient to induce localized damage leading to product rejection, even though the metal is not externally constrained during solidification. This phenomenon, often termed hot tearing or hot cracking, is an important defect in a range of processes from shape casting $^{[1]}$ to welding, ${ }^{[2]}$ but is poorly understood.

Over the years, a number of researchers have experimentally investigated hot tearing. Pellini ${ }^{[3]}$ was the first author to demonstrate that hot tears form in the semisolid. Feurer ${ }^{[4]}$ examined the influence of alloy composition and solidification conditions on hot tearing, and proposed that this defect was a result of the inability of liquid to feed solidification shrinkage. Warrington and McCartney ${ }^{[5]}$ examined the effect of grain refining on hot tearing, and found that hot tears formed easily in columnar and equiaxed-globular grain structures, but not in equiaxed-dendritic structures. Using experimental data from ring castings, Guven

A.B. PHILLION, formerly Doctoral Candidate, Department of Materials Engineering, University of British Columbia, is Postdoctoral Fellow, Computational Materials Laboratory, Institute of Materials, Ecole Polytechnique Fédérale de Lausanne, Lausanne, CH 1015, Switzerland. Contact e-mail: andre.phillion@epfl.ch P.D. LEE, Professor, is with Department of Materials, Imperial College London, Prince Consort Road, London, SW7 2BP, United Kingdom. E. MAIRE, Research Associate, is with Université de Lyon, INSALyon, Villeurbanne 69621, France. S.L. COCKCROFT, Professor and Department Head, is with Department of Materials Engineering,

University of British Columbia, Vancouver, Canada V6T 1 Z4.

Manuscript submitted January 21, 2008.

Article published online July 15, 2008 and $\mathrm{Hunt}^{[6]}$ showed that hot tears initiate in a thin film of liquid between two grains. While these studies have proved insightful in developing processes that are less susceptible to hot tearing, they lack insight into the underlying mechanisms controlling hot tear formation.

To examine these underlying mechanisms, a few authors have designed experiments that allow for observation of semisolid crack initiation. Pellini ${ }^{[3]}$ used $\mathrm{X}$-ray radiography to make the first observations of hot tear formation in aluminum-copper alloys. Fredriksson and Lehtinen ${ }^{[7]}$ performed hot tensile tests inside a scanning electron microscope, showing that hot cracks occur if the alloy contains a eutectic liquid with good ability to wet the solid grain boundaries. Davidson et al. ${ }^{[8]}$ recorded the formation of hot tears in an aluminum-copper alloy during solidification using a video camera and determined that hot tearing begins to occur with very small applied loads at fraction solids between 0.93 and 0.96. Farup et al. ${ }^{[9]}$ used an organic analogue, succinonitrile-acetone, to directly observe the nucleation of hot tears. While these in-situ tests have been revealing, they have been limited by a number of factors. First, in each experiment the observation of hot tears is limited to those appearing on the free surface. Thus, in the metallic studies, the initiation of the hot tear is probably missed. Second, the correlation between an organic analogue and a metal has not been demonstrated, since differences exist between the two systems (e.g., the formation of last eutectic and oxides in aluminum alloys). Third, the mechanisms controlling hot tear formation (e.g., void nucleation and growth) have been difficult to observe due to the metal opacity.

The process of void formation leading to hot tearing is complex and poorly understood. One recent study designed to identify the underlying mechanisms was performed by Farup et al. ${ }^{[9]}$ which allowed for observation of three different mechanisms for hot tear nucleation: "1. directly as elongated pores or tears, 2 . on pores caused by solidification shrinkage, or 3. as round pores nucleated in the liquid constituting a healed 
hot tear." Thus, it is clear that in the early stages, hot tearing is controlled by the formation and growth of internal voids. In a second recent study, Fredriksson et $a l^{[10]}$ presented a thermodynamic description of hot tear nucleation. In this work, it was proposed that hot tear nucleation is enhanced by the supersaturation of vacancies, since these vacancies will cluster to form voids at grain boundaries. After nucleation, hot tear growth would occur by a combination of vacancy diffusion and also by the decrease in free energy when stored elastic energy from thermal and mechanical stress is released as crack growth. Void nucleation in semisolid metals has also been investigated. Piwonka proposed that dissolved gases aid in void nucleation. ${ }^{[11]}$ Campbell ${ }^{[1]}$ has shown that the cavitation pressure required to form voids is large in comparison to the expected shrinkage pressure drop during solidification. Instead, it was proposed that voids form via separation of the solid-liquid interface and at entrained oxides or other heterogeneous nuclei. Hirth et al. ${ }^{[12]}$ proposed a thermodynamic analysis to describe the rate of void nucleation due to cavitation, boiling, and degasification processes. However, in all these cases, the initial size of these nucleating voids was not discussed. Nor was any relationship to semisolid microstructure or stress state presented. Both of these features are important relationships for understanding the underlying mechanisms of hot tear nucleation.

In this study, X-ray microtomography (XMT) is performed on interrupted semisolid tensile tests to quantify the evolution of internal damage in three dimensions and thus to develop insight into the early and late stages of hot tearing. This methodology overcomes the problems of earlier two-dimensional (2-D) surface observations, and allows for observation of the microstructural features relevant to hot tear formation.

\section{EXPERIMENTAL METHODOLOGY}

The experimental approach employed for the investigation involved the following three steps: (1) partially remelting a previously cast aluminum alloy specimen, (2) deforming the specimen in tension, and (3) performing XMT on the portion of the gage length where damage localization occurred. The tensile tests can be described as "interrupted" tests, since each test was stopped during the deformation process to conduct an off-line tomography scan before reheating and then continued application of load. The semisolid deformation tests were conducted at The University of British Columbia, while the tomographic data was collected at Imperial College.

\section{A. Materials and Geometry}

A commercially direct chill (DC) cast aluminum AA5182 rectangular ingot, of composition Al-4.63 pet $\mathrm{Mg}-0.49$ pet $\mathrm{Mn}-0.17$ pet $\mathrm{Fe}-0.04$ pet $\mathrm{Cu}$, was chosen as the source for the as-cast specimens. Although the hydrogen content of the specimens is unknown, the ingot was industrially degassed during the commercial casting process. The semisolid constitutive behavior of this alloy has been previously characterized, by both Colley et al. ${ }^{[13]}$ and Van Haaften et al. ${ }^{[14]}$ Colley's work showed that the alloy exhibits tensile strength up to a temperature of $\sim 575^{\circ} \mathrm{C}$, corresponding to a fraction solid of $\sim 0.95$, and exhibits some tensile ductility up to a temperature of $\sim 565{ }^{\circ} \mathrm{C}$. Note that since the material is in the as-cast state, it is not damage free and has been subjected to a strain history associated with the DC casting process.

Tensile specimens of $100 \mathrm{~mm}$ in gage length and $4.0 \mathrm{~mm}$ in radius, shown in Figure 1, were machined out of the ingot with their long axis orientated normal to the casting direction and parallel to the broad face of the ingot. They were extracted from material 60 to $100 \mathrm{~mm}$ below the surface of the ingot. This orientation was chosen such that deformation in the specimens takes place in the same direction and in the same region as the occurrence of hot tearing in ingot DC casting. The central $10 \mathrm{~mm}$ of each specimen was further reduced in radius to $3.5 \mathrm{~mm}$ to ensure that the thermal hot-spot and corresponding strain occurred at a known location. A total of three specimens of this format were prepared for testing. Three small cylinders were also prepared for testing; two out of the as-cast ingot $(\phi=2.0 \mathrm{~mm}$ and $1.6 \mathrm{~mm}$, where $\phi$ is the specimen diameter) and the third $(\phi=2.0 \mathrm{~mm})$ from one of the tensile specimens following deformation.

\section{B. Semisolid Deformation}

The semisolid deformation tests were performed using a previously developed mushy zone tensile tester (MZTT). ${ }^{[13]}$ This apparatus consists of a modified Instron (Instron Inc, Norwood, MA) mechanical testing machine connected to a Gleeble 3500 (Dynamic Systems Inc., Poestenkill, NY) thermomechanical simulator for rapid $I^{2} R$ resistive heating of the specimen. The temperature chosen for semisolid deformation was $528^{\circ} \mathrm{C}$. This temperature corresponds to a fraction solid of approximately $\sim 0.98,{ }^{[15]}$ and was chosen based on the authors' prior experience with this alloy. ${ }^{[16]}$

The experimental campaign is shown in Table I. Prior to hot deformation, each specimen was subjected to a tomographic scan to characterize the initial as-cast void distribution (subscript " 0 " in Table I). Specimens were then heated at a rate of $1.5^{\circ} \mathrm{C} \mathrm{s}^{-1}$ using the MZTT until the test temperature was reached. Semisolid deformation was subsequently applied, at a displacement rate of $0.085 \mathrm{~mm} \mathrm{~s}^{-1}$, to create internal damage. After $\sim 12$

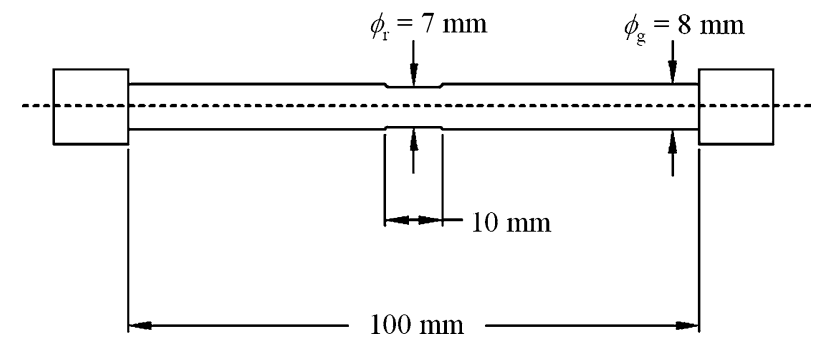

Fig. 1-Interrupted tensile test specimen geometry, showing reduced area region. 
Table I. Specimen Text Matrix and Results of the XMT Analysis Performed at a Voxel Size of $9 \mu \mathrm{m}$

\begin{tabular}{|c|c|c|c|c|c|c|}
\hline Specimen & $\begin{array}{c}\text { Test } \\
\text { Level }\end{array}$ & $\varepsilon_{\mathrm{tot}}$ & $\varepsilon_{d}$ & $\begin{array}{l}\text { Percent } \\
\text { Porosity }\end{array}$ & $\begin{array}{c}N_{v} \\
\left(\mathrm{~mm}^{-3}\right)\end{array}$ & $\begin{array}{l}\text { Maximum } \\
\text { Void Size } \\
\quad(\mu \mathrm{m})\end{array}$ \\
\hline \multirow[t]{3}{*}{$A$} & $A_{0}$ & 0 & 0 & 0.52 & 26 & 113 \\
\hline & $A_{1}$ & 0.09 & 0.06 & 3.22 & 38 & 391 \\
\hline & $A_{2}$ & 0.39 & 0.20 & 16.49 & 60 & 1108 \\
\hline \multirow[t]{3}{*}{$B$} & $B_{0}$ & 0 & 0 & 0.26 & 8 & 99 \\
\hline & $B_{1}$ & 0.11 & 0.09 & 1.92 & 76 & 173 \\
\hline & $B_{2}$ & 0.39 & 0.25 & 12.55 & 49 & 1012 \\
\hline \multirow[t]{2}{*}{$C$} & $C_{0}$ & 0 & 0 & 0.74 & 16 & 188 \\
\hline & $C_{1}$ & 0.32 & 0.18 & 13.07 & 94 & 1012 \\
\hline
\end{tabular}

seconds loading time, the test was interrupted and the specimen was cooled and removed from the MZTT to perform a tomographic scan on the material deformed to the first displacement level (subscript 1 in Table I). This procedure was repeated to create further internal damage in the reduced gage region (subscript 2 in Table I). Note that specimen $C$ did not undergo the second level of displacement application since it appeared to be heavily damaged following the first displacement level $C_{1}$.

One feature of the apparatus is that a parabolic temperature gradient prevails along the specimen, promoting strain localization near the center of the gage length. This arises due to both conduction of heat to the water-cooled copper grips, and the increased $I^{2} R$ heating in the reduced gage region. The addition of the reduced gage region, containing the control thermocouple, ensured that the hot spot and thus strain localization occurred at the same location for both displacement levels.

\section{Tomographic Imaging}

X-ray microtomography was performed on the reduced gage region of each specimen using a commercial laboratory-scale XMT unit (Phoenix $\mid X$-ray Systems and Services GmbH, Wunstorf, Germany). To fully capture the entire reduced gage region, three successive subscans were performed at a voxel resolution of $9 \mu \mathrm{m}$. The resolution was constrained by the diameter of the tensile specimens. For each scan, 720 radiographs, scanning 360 $\mathrm{deg}$, were taken along the loading axis at $0.5-\mathrm{deg}$ increments and at three different $z$-positions. X-ray microtomography scans using the same XMT unit were also performed on the two 2.0 -mm-diameter specimens to conduct high-resolution tomographic scans on both the initial as-cast and deformed states (prepared from sample $C_{1}$ ), at a voxel resolution of $2.5 \mu \mathrm{m}$. Image slices were reconstructed from the series of projections based on the filtered back-projection method, ${ }^{[17]}$ to create digital volumes consisting of $850 \times 850 \times 1100$ voxels at a 16-bit floating point grayscale range. A single tomography scan was also performed on the 1.6-mm-diameter ascast specimen at the European Synchrotron Radiation Facility (ESRF), beamline ID19, at a voxel resolution of $0.7 \mu \mathrm{m}$. Further details on the ESRF testing methodology can be found in References 18 through 20.

\section{Analysis of the Tomographic Data}

To evaluate the microstructural effects quantitatively, the eight reconstructed tomographic datasets of the entire reduced gage region and the three high-resolution datasets were subjected to image analysis using the software packages Image $\mathrm{J}^{[21]}$ and Amira (Mercury Computer Systems, Chelmsford, MA). A series of filters were applied to each dataset to enhance the contrast between void, metal, and exterior. First, the full dataset was transformed from 16-bit to 8-bit grayscale to reduce the size of the digital file. Second, a circle that fits the exterior surface was drawn on each of the image slices. Voxels outside this circle were given a grayscale value of 255, while voxels inside this circle remained at their original value (between 0 and 254). This reclassified all external voids, i.e., voids that interfaced both metal and the exterior, as internal voids. Third, an edge-preserving-smoothing filter was applied to the full dataset to remove noise in both the metal and void regions while preserving the metal-void boundaries. Further details on this filter can be found in Reference 22. Finally, a threshold was imposed on the dataset to explicitly label each voxel as void, metal, or exterior. Unfortunately, it was not possible to follow individual voids during deformation because the rotational alignment of the specimen with respect to the X-ray detector in the XMT unit was not consistent between the tomographic scans.

It was observed in the datasets for specimens $A$ and $B$, taken after the second level of semisolid deformation, that most of the damage was contained within a small portion ( $2 \mathrm{~mm}$ in longitudinal direction) of the reduced gage region. A child volume of this region was cropped out of the full dataset, and child volumes of the identical region were cropped out of the datasets taken from the as-cast and the first level of semisolid deformation for all four specimens. The child dataset was then relabeled with each void assigned a different grayscale value, allowing for the volume of each void to be determined. Furthermore, the average external diameter was calculated based on the series of circles which were fit to the exterior surface in each image slice (discussed previously), while the percentage porosity was calculated based on the total volume of voids found in the child dataset.

\section{RESULTS AND DISCUSSION}

Analysis of the tomographic data allowed for both qualitative and quantitative comparisons of the damage formed during semisolid tensile deformation as a function of strain. In this work, two definitions of strain have been used

$$
\begin{gathered}
\varepsilon_{d}=2 \cdot \ln \left(\frac{d_{0}}{d_{i}}\right) \\
\varepsilon_{\mathrm{tot}}=\ln \left(\frac{\pi\left(d_{0} / 2\right)^{2}}{\left(\sum \mathrm{voxels}_{i} \cdot A_{\mathrm{vox}}\right.}\right)
\end{gathered}
$$

where $\varepsilon_{\mathrm{d}}$ is the strain estimated based on the average external diameter, $d_{\mathrm{i}}$ at each level of deformation 
( $i=0,1,2)$, whereas $\varepsilon_{\text {tot }}$ is the strain estimated based on the number of voxels representing metal in each slice of the reconstructed datasets ( $\sum$ voxels), and $A_{\text {vox }}$ is the cross-sectional area of each voxel. $\varepsilon_{d}$ thus takes into account the accumulation of internal damage during deformation.

\section{A. Qualitative Assessment}

The evolution of damage as a function of strain during semisolid tensile deformation of as-cast AA5182 is shown qualitatively in Figures 2 and 3. The images shown are from specimen $A$, and are typical of the results seen in all three specimens. In Figure 2, a 2-D cross-sectional image of the three-dimensional (3-D) tomographic data at each strain level is shown, with the loading direction normal to image. The dark areas represent voids, while the small bright white areas represent solute phases. In Figure 3, the 3-D morphology of a quarter section of the internal damage/void network at each strain level is presented via a threshold and segmentation process. This morphology is thought to play an important role in the final formation of macroscopic hot tears. Two symmetry planes and a cross-sectional slice are also displayed in the background of Figure 3 to provide an indication of the specimen external dimensions. Note that in both Figures 2 and 3, the same location within the specimen is shown but at three different levels of deformation.

Figure 2(a) shows the initial as-cast state of specimen $A$, which contains 0.52 pct porosity, evenly distributed throughout the cross section. After the material has been deformed in the semisolid state to a total strain $\varepsilon_{\text {tot }}$ of 0.09 (Figure 2(b)), the amount of internal damage has increased substantially to 3.22 pct. With a further increase in semisolid deformation to a total strain $\varepsilon_{\text {tot }}$ of 0.39 (Figure 2(c)), the internal damage has become quite extensive and localized.

Figure 3(a) shows the initial distribution of voids in specimen $A$ in three dimensions. As can be seen from the figure, the voids are quite tortuous and their maximum length is much longer than is apparent from the 2-D cross section (Figure 2(a)). In the early stages of semisolid deformation, (Figure 3(b)), it appears that both discrete growth of the pre-existing voids and formation of new voids has occurred. It is unclear whether the formation of new voids is a consequence of void nucleation, or the rather large voxel size of $9 \mu \mathrm{m}$. With the large voxel size, small voids which were below the resolution limit in Figure 3(a) may have simply grown to the point where they are now large enough to be detected by the tomography scan. The results from the tomography scan performed at ESRF, at a voxel size of $0.7 \mu \mathrm{m}$, showed that the as-cast AA5182 material contains a void number density of $\sim 11,000 \mathrm{~mm}^{-3}$. These well-distributed and small voids, shown in Figure 4(a), could easily grow to accommodate strain without the material resorting to void nucleation, and thus appear as new voids in Figure 3(b). Unfortunately, the small voxel size at ESRF misses the larger voids and also misses hot tears due to the tiny field of view of $0.875 \mathrm{~mm}^{3}$ in volume. Note that the implications of using voxel sizes
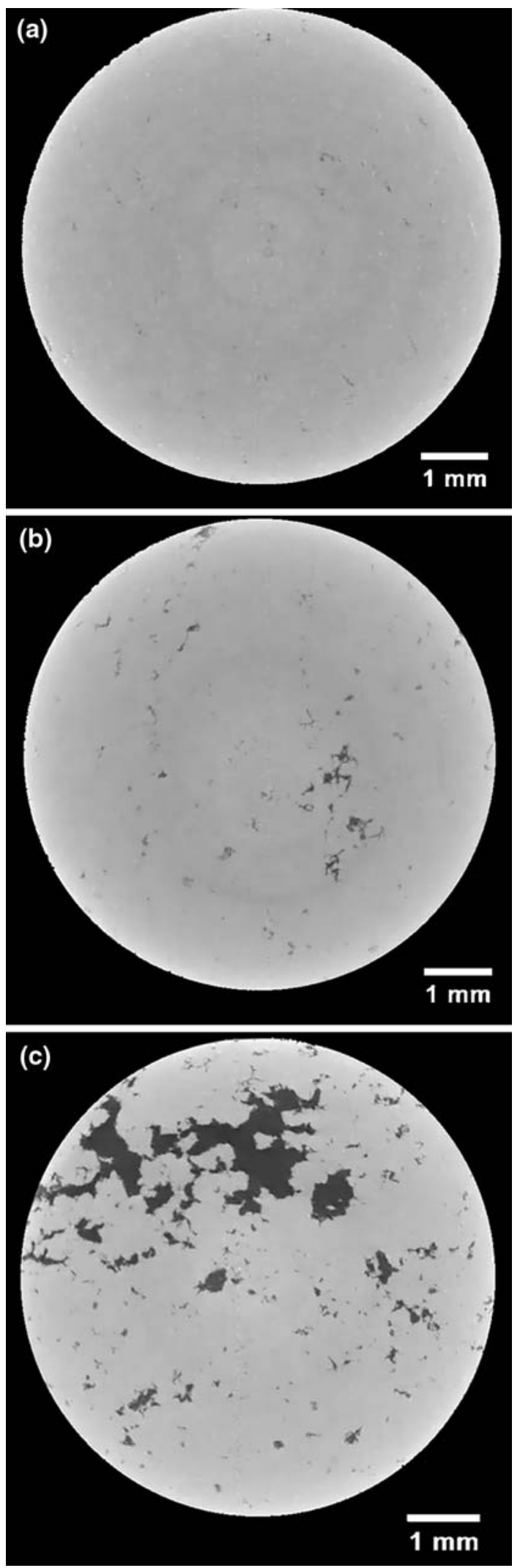

Fig. 2-Transverse sections from XMT scans of specimen $A$ showing initial porosity in the specimen, and then development of further damage with application of strain: $(a) \varepsilon_{\text {tot }}=0$, pct $P=0.52\left(A_{0}\right)$; (b) $\varepsilon_{\mathrm{tot}}=0.09$, pct $P=3.22 \quad\left(A_{1}\right) ;$ and $(c) \varepsilon_{\mathrm{tot}}=0.39$, pct $P=16.49\left(A_{2}\right)$. The XMT scan resolution was $9 \mu \mathrm{m}$ per voxel. 


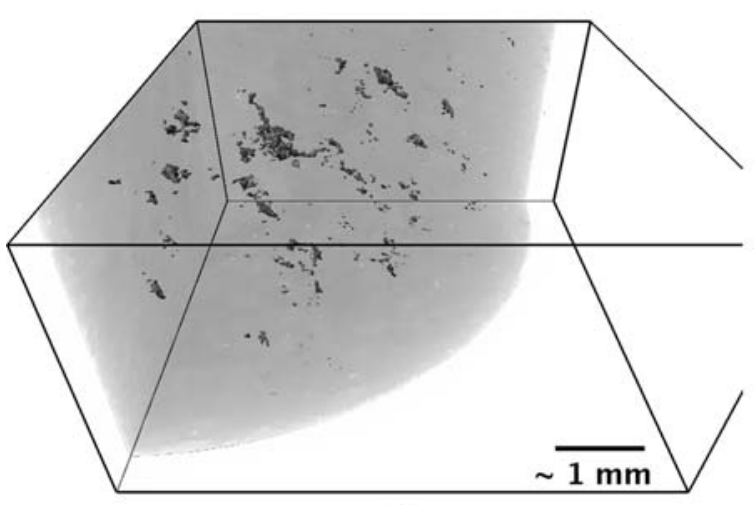

(a)

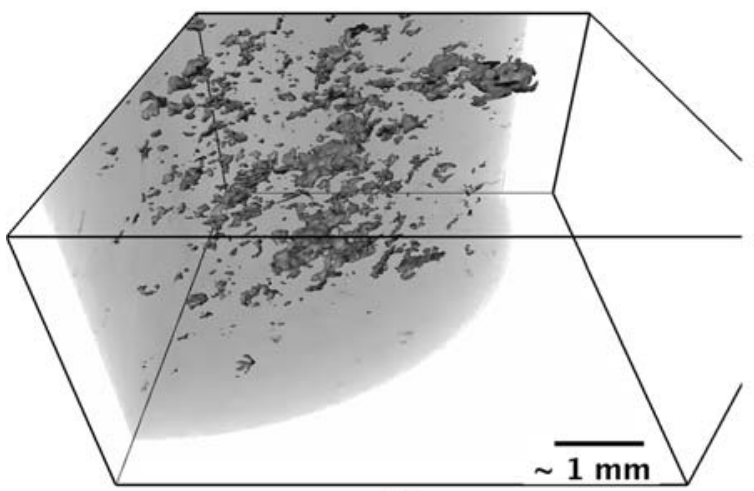

(b)

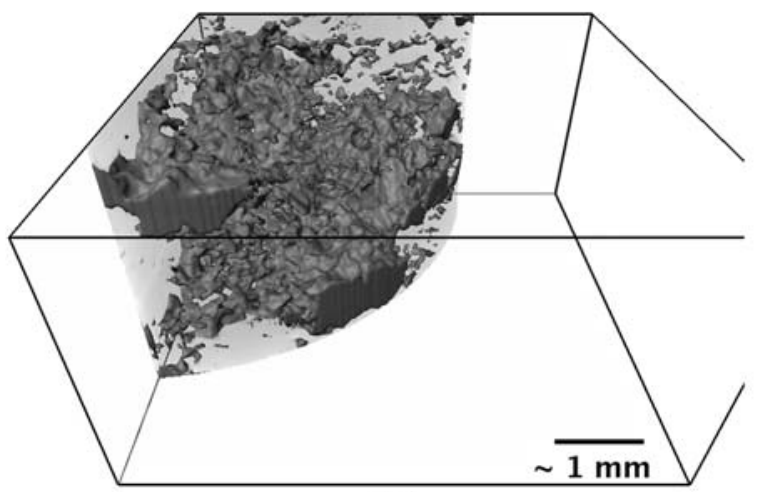

(c)

Fig. 3-3-D morphology of the internal damage in specimen $A$ observed by XMT at various levels of strain in a quarter section of the deformed region at strain levels of $(a) \varepsilon_{\text {tot }}=0$, pct $P=0.52$ $\left(A_{0}\right) ;(b) \varepsilon_{\mathrm{tot}}=0.09$, pct $P=3.22\left(A_{1}\right) ;$ and $(c) \varepsilon_{\mathrm{tot}}=0.39$, pct $P=16.49\left(A_{2}\right)$. The XMT scan resolution was $9 \mu \mathrm{m}$ per voxel.

of $9,2.5$, and $0.7 \mu \mathrm{m}$ for the various tomography scans are discussed in terms of spatial resolution in Section III-B. Also, the current ESRF tomography scan results correlate well with the study by Maire et al., ${ }^{[23]}$ who previously quantified the distribution of voids in as-cast AA5182 via tomography at ESRF. This previous study found a void number density of $\sim 2025 \mathrm{~mm}^{-3}$, which is lower than the current value but may have been calculated using a larger void size as a threshold value. Furthermore, the effects of specimen location within the ingot, as well as the general casting conditions on the formation of the very small as-cast pores may be important. With a further increase in strain (Figure 3(c)), the void morphology has now become a highly complex, localized, and interconnected network of internal damage.

Figures 2 and 3 provide new insight into the extent of void formation, growth, and coalescence occurring during semisolid deformation processes. The as-cast porosity seems to play an important role, acting as preexisting nuclei for void growth. As strain is applied, the growing voids appear to be preferentially orientating toward each other facilitating their eventual coalescence. Unfortunately, the $9 \mu \mathrm{m}$ voxel size of the images shown in Figures 2 and 3 is too large to indirectly observe the role of the liquid in void formation, growth, and coalescence.

To investigate liquid/void interaction, tomographic datasets of the initial as-cast material and a small portion of specimen $C_{1}$ were obtained at a voxel size of $2.5 \mu \mathrm{m}$. The 2-D cross-section images of these tomography scans are shown in Figures 4(b) and (c). The higher resolution allows for observation of some salient features. First, small bright white areas are clearly visible in Figures 4(b) and (c), and represent the $\mathrm{Mn} / \mathrm{Fe} /$ $\mathrm{Cu}$ enriched eutectic and intermetallic phases. Although the grain boundaries and triple points are not visible, their location is marked by these secondary phases. ${ }^{[24]}$ Second, the as-cast porosity appears to be located at the grain boundaries, as previously illustrated by Lee and Hunt. ${ }^{[2]}$ The grain size was measured to be $\sim 225$ to $250 \mu \mathrm{m}$ using optical metallography of anodized specimens and correlates well to the distance between the white phases in Figure 4(b). Finally, it may be hypothesized that the deformation-based voids seen in Figure 4(c) formed near the triple points, since voids (1), (2), and (3) are linked by relatively small channels surrounded by the bright white secondary phases. These small channels may have been grain boundaries or interdendritic eutectic, and thus liquid during semisolid deformation. Note that the tail of material in both images is due to the process of wire electric discharge machining, which was used to make the cylinders.

\section{B. Quantitative Assessment}

The 3-D image-analysis software allows for calculation of the volume of individual voids in the deformed region of each specimen. The bulk porosity, void number density, and the maximum void size at different levels of strain for specimens $A, B$, and $C$ is shown in Table I. The variation in the distribution of void radii for different values of strain for specimens $A, B$, and $C$ is shown in Figures 5 through 8 . These figures are plotted such that the data displayed have been divided into 15 different bins on a $\log$ scale. In Table I and Figures 5 through 8 , the voxel size was $9 \mu \mathrm{m}$.

Beginning with the as-cast material, it can be seen from Table $I$ that there is a wide range in the initial porosity level $(0.26<$ pct $P<0.74)$, the maximum initial void radius $(99 \mu \mathrm{m}<r<188 \mu \mathrm{m})$, and also the initial void number density $\left(8 \mathrm{~mm}^{-3}<N_{v}<26 \mathrm{~mm}^{-3}\right)$ in the three specimens. These initial voids are most 

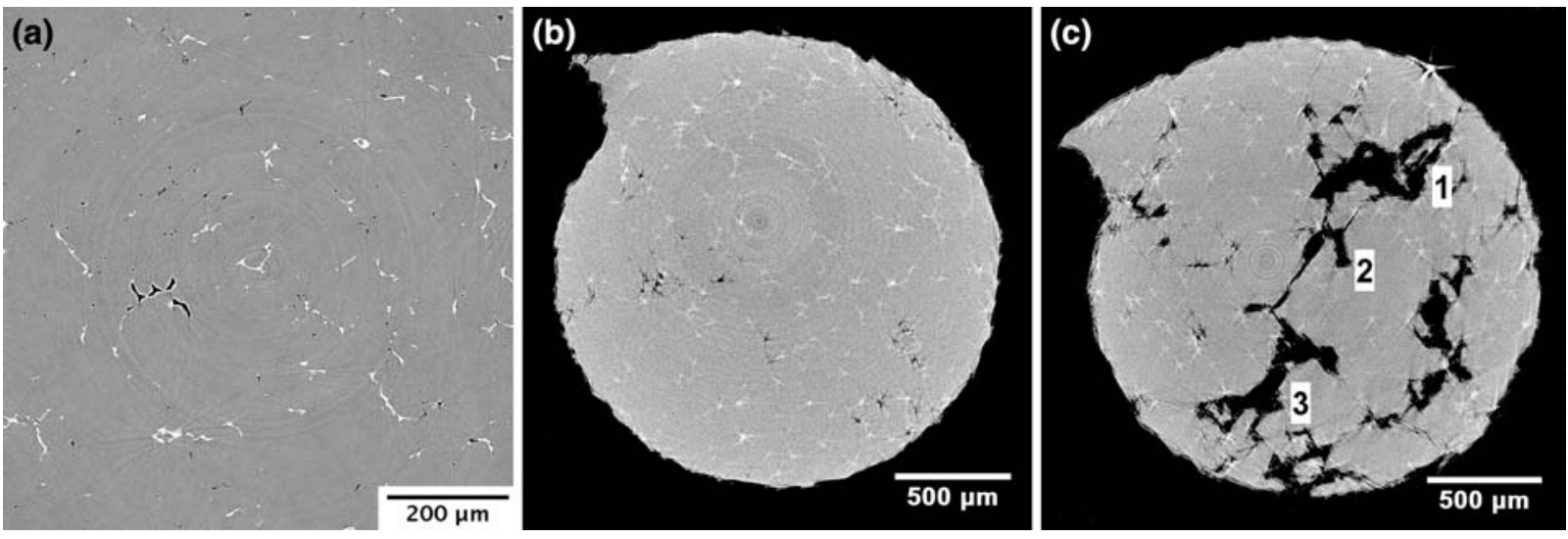

Fig. 4-Transverse sections from high-resolution XMT scans of (a) as-cast AA5182 (ESRF, voxel size of $0.7 \mu \mathrm{m}$ ); (b) as-cast AA5182 (Lab XMT, voxel size of $2.5 \mu \mathrm{m})$; and $(c)$ specimen $C_{1}, \varepsilon_{\text {tot }}=0.32$ (Lab XMT, voxel size of $\left.2.5 \mu \mathrm{m}\right)$. Black areas represent voids, while white areas represent $(\mathrm{Mn}, \mathrm{Fe})$ enriched solute phases.

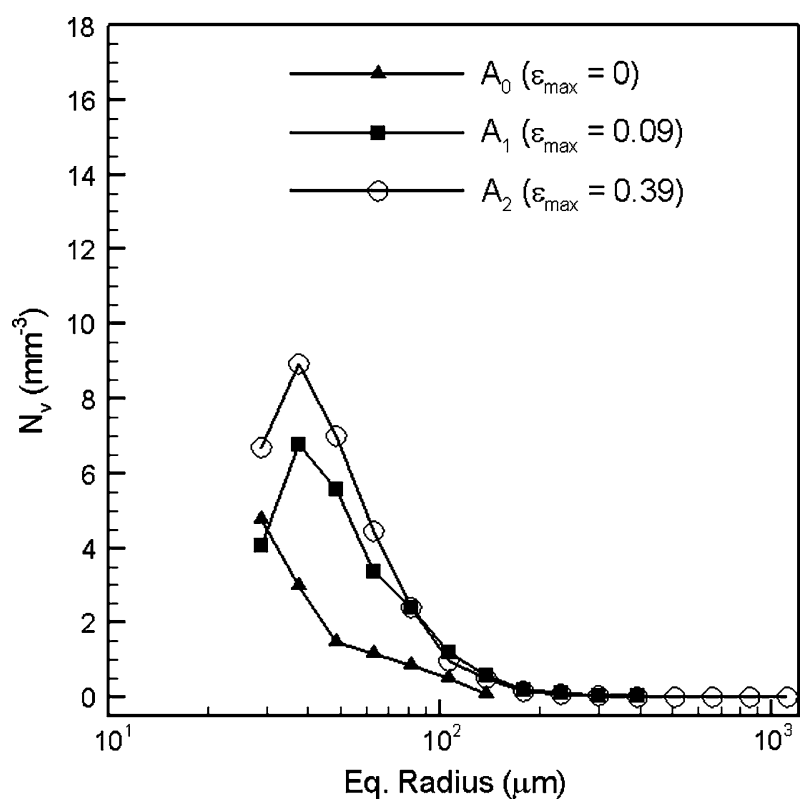

Fig. 5-Number density of voids in specimen $A\left(A_{0}, A_{1}\right.$, and $\left.A_{2}\right)$ as a function of equivalent radius.

probably a combination of shrinkage and hydrogenbased porosity ${ }^{[26,27]}$ and may also have been caused or augmented by some strain accumulated during the casting process. Since all three specimens were subjected to similar local solidification conditions, the observed variation in as-cast porosity is most probably due to small variations in composition (especially hydrogen), feeding conditions, and potential nuclei such as inclusions, e.g., oxides. These secondary particles may enhance void nucleation in specific locations and allow large voids to form. The variation in as-cast porosity will have a large effect on the nucleation and localization of damage, and hence on the semisolid deformation behavior.

As shown in Table I, the level of porosity, maximum void radius and void number density increase significantly with semisolid tensile deformation. One interesting

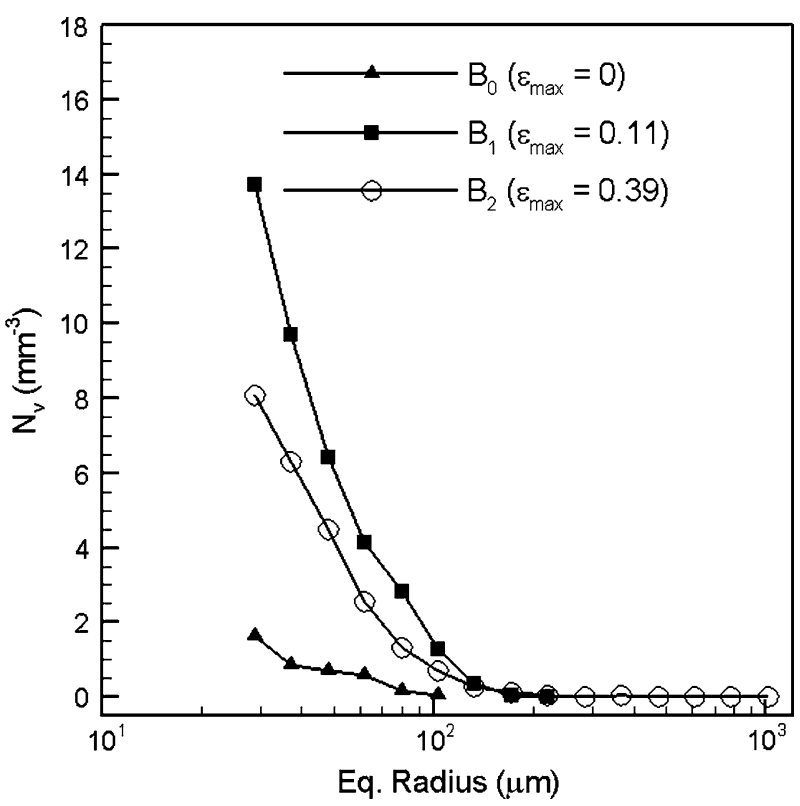

Fig. 6-Number density of voids in specimen $B\left(B_{0}, B_{1}\right.$, and $\left.B_{2}\right)$ as a function of equivalent radius.

observation is the interaction between dimetral reduction and internal damage. As would be expected due to conservation of volume, the specimen diameter is decreasing with increasing deformation. During solid ductile yielding, small voids only develop after significant yielding, leading to strain localization. In contrast, these experiments show that in semisolid deformation, a different sequence occurs in which significant internal damage develops to accommodate the deformation. For example, the dimetral strain $\varepsilon_{d}$ for specimen $A_{2}$ was 0.25 , while the total strain $\varepsilon_{\text {tot }}$ was 0.39 . Thus, the internal damage accounts for approximately one-third to one-half of the total strain obtained during deformation. In order to properly characterize semisolid constitutive behavior, this internal damage must also be included.

The variation in the number density of voids as a function of equivalent void radius for the as-cast and 


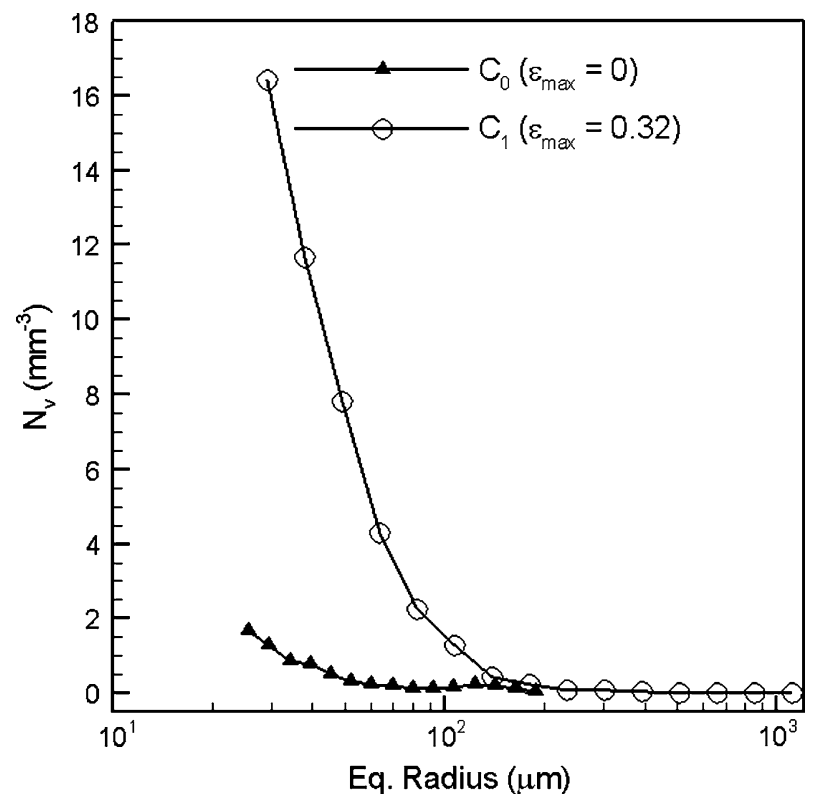

Fig. 7-Number density of voids in specimen $C\left(C_{0}\right.$ and $\left.C_{1}\right)$ as a function of equivalent radius.

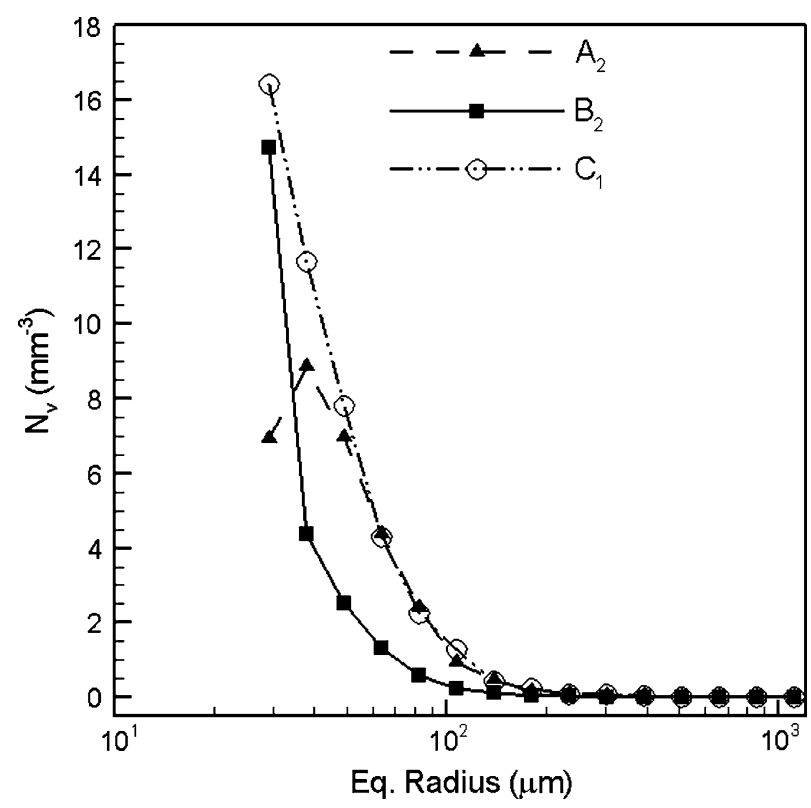

Fig. 8 - Comparison of number density of voids in $A_{2}, B_{2}$, and $C_{1}$ (the three failed specimens, $\varepsilon \sim 0.35$ ), as a function of equivalent radius.

semisolid deformed states is presented in Figures 5 through 7 for specimens $A, B$, and $C$. To determine an appropriate spatial resolution for this quantification, the computed number density of voids as a function of equivalent radius observed in $A_{0}, B_{0}$, and $C_{0}$ was compared to the values obtained for undeformed material scanned at higher resolution using both the commercial laboratory-scale XMT unit at a voxel size of $2.5 \mu \mathrm{m}$ and the ESRF tomography apparatus at a voxel size of $0.7 \mu \mathrm{m}$. The three resolutions provided a similar void number density only for void radii greater than $\sim 25 \mu \mathrm{m}$. Below this value, however, far more small voids were found in both of the higher-resolution scans as compared to specimens $A, B$, and $C$. The void radius $25 \mu \mathrm{m}$ corresponds to a cube of dimensions $4.45^{3}$ voxels and thus voids occupying a volume less than $4^{3}$ voxels were excluded from the quantitative analysis. Similarly, far more voids were found in the tomography scan performed at ESRF as compared to the commercial laboratory-scale scan performed at a voxel size of $2.5 \mu \mathrm{m}$. However, the results from the ESRF scan do not describe the variation in computed void density well for larger voids. This, together with the sample diameter spanning only a few grains, means that the very small samples required for the high resolution at ESRF are inappropriate for MZTT hot tear analysis.

The number density of voids as a function of equivalent void radius for specimen $A$ is shown in Figure 5. As can be seen in the figure, the first semisolid deformation $\left(A_{1}\right)$ has resulted in both the appearance of many new small voids and the growth of a few voids. The largest void was $391 \mu \mathrm{m}$ in equivalent radius. As previously discussed, the large number of small voids observed in the ESRF data suggests that most of the new voids are due to the growth of voids that occupied a volume less than $4^{3}$ voxels in the initial scan $A_{0}$, and not void nucleation. Continued deformation to level $A_{2}$ results in further void formation, and significant void growth and coalescence, with one void being $1012 \mu \mathrm{m}$ in equivalent radius. Note that in curves $A_{1}$ and $A_{2}$, the first bin has a lower value for void number density than the second bin. This apparent peak value in the curve is thought to be due to the spatial resolution limit of $4^{3}$ voxels, rather than an actual peak value in the void number density distribution.

The number density of voids as a function of equivalent void radius for specimen $B$ is shown in Figure 6. These results are quite different as compared to specimen $A$ and indicate that after the first semisolid deformation $\left(B_{1}\right)$, the maximum number density in bin one, containing the smallest size range, has risen from $2 \mathrm{~mm}^{-3}$ to over $14 \mathrm{~mm}^{-3}$, and the total number density of voids has increased by $\sim 850$ pct. After the second semisolid deformation $\left(B_{2}\right)$, some very large voids were found in the deformed region, and the total number density of voids had decreased by $\sim 45$ pct (as compared to $B_{1}$ ). The large increase in the number density of voids from $B_{0}$ to $B_{1}$ supports the view that a portion of the newly detected voids results from void nucleation processes and not exclusively growth of small as-cast voids, while the decrease in total void number density from $B_{1}$ to $B_{2}$ clearly indicates that void coalescence was occurring during the second increment in strain. In contrast, in specimen $\mathrm{A}$, there was a continuous increase in the void number density with increasing strain: from $\sim 4 \mathrm{~mm}^{-3}\left(A_{0}\right)$ to $\sim 6.5 \mathrm{~mm}^{-3}\left(A_{1}\right)$ to $\sim 9.5 \mathrm{~mm}^{-3}\left(A_{2}\right)$.

The number density of voids as a function of equivalent void radius for specimen $C$ is shown in Figure 7. As can be seen in this figure, there has been a substantial increase in the number of small voids since the maximum number density in bin one, containing the smallest size range, has risen from $2 \mathrm{~mm}^{-3}$ to over 
$16 \mathrm{~mm}^{-3}$ during the first semisolid deformation $\left(C_{1}\right)$. Furthermore, there must have been void growth and coalescence since large voids also exist, with radii of 508, 660, 854, and $1108 \mu \mathrm{m}$.

On a macroscopic level, the semisolid deformation imparted to $A_{2}, B_{2}$, and $C_{1}$ is quite similar in terms of both total strain and diametral strain. In Figure 8 , the variation in the number density of voids as a function of equivalent void radius is shown for $A_{2}, B_{2}$, and $C_{1}$. As can be seen from the figure, the structure that results from applying these levels of deformation to a semisolid material contains many small and medium sized internal voids. Only a few voids greater than $225 \mu \mathrm{m}$ have formed ( $<5$ in each bin), although they contribute to creating most of the internal damage. The similarity in the shape of the curves for all three specimens suggests that the same damage mechanisms were in play. However, there were important differences in their behavior, as shown in Figures 5 through 7 and Table I. For example, there was a continuous increase in the void number density with increasing strain in specimen $A$, while there was clear evidence of void coalescence in specimen $B$, since the total number of voids decreased sharply between the first and second levels of deformation. In the case of specimen $C$, the material accumulated significantly more damage and strain (both diametral and total strain) following the first level of deformation as compared to specimens $A$ and $B$. It is hypothesized that this difference occurs due to the initial state of the material, since specimen $C$ initially contained a few large as-cast pores (e.g., $r_{e q}=162,172$, and $188 \mu \mathrm{m}$ ), which were not present in the other two specimens. These larger voids would have allowed growth and coalescence processes to activate much earlier. Thus, it appears that the as-cast porosity has a significant effect on hot tearing susceptibility.

The qualitative and quantitative analyses of strainassisted void development have shown that the semisolid deformation process is controlled by discrete growth and coalescence of voids. In the deformed region of each of the specimens, significant amounts of strain have been accommodated by internal damage accumulation in addition to dimetral reduction. At some critical strain, localized void coalescence occurs, which dominates damage accumulation. This was shown in specimen $B$ (Figure 6), with the decrease in void number from the first to the second round of semisolid deformation.

\section{Internal Damage Spatial Orientation}

To further understand the mechanisms of semisolid deformation, the spatial orientation of each void relative to the loading direction was examined. This spatial orientation refers to the direction cosine of each void's major axis, termed the morphological texture. The results for specimen $A$ at strain levels $A_{0}, A_{1}$, and $A_{2}$ are presented in a series of equal-area pole figures similar to a texture plot for grain orientation (Figure 9). The loading direction is perpendicular to the pole figure, with points at $(0,0)$ corresponding to voids having a major axis parallel to the loading direction, and points on the circle's perimeter corresponding to a major axis perpendicular to the loading direction. The major axis of each void was calculated from the eigenvectors of a covariance matrix based on the $x, y$, and $z$ coordinates of all voxels in that void. The eigenvectors were determined using the principal component analysis technique, ITK libraries, ${ }^{[28]}$ and in-house coding. Note that to reduce noise, only the results for voids containing more than 1000 voxels $\left(10^{3}\right)$ are reported.

The analysis of as-cast porosity (Figure 9(a)) shows that on average their major axis is located in one plane. This bias is thought to arise due to the direction of progress of the solid-liquid interface during DC casting. With increasing strain (Figure 9(b)), the morphology has evolved considerably. At this moderate level of strain, the voids seem to be growing in all directions as compared to the loading direction, since the major axes are scattered throughout the pole plot. Furthermore, the number of voids exceeding the size threshold has also increased significantly due to void growth. At higher strain (Figure 9(c)), it is clear that almost all voids are becoming preferentially oriented perpendicular to the

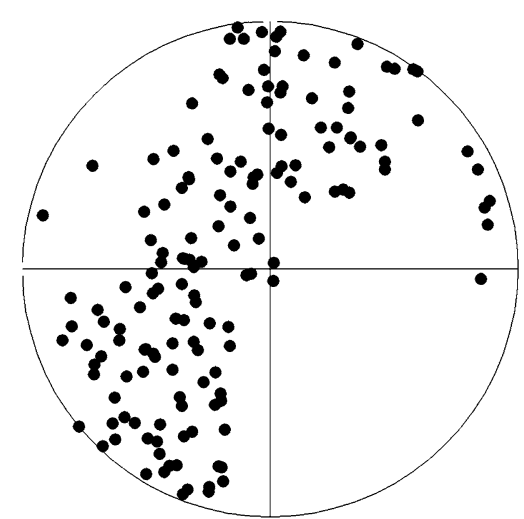

(a)

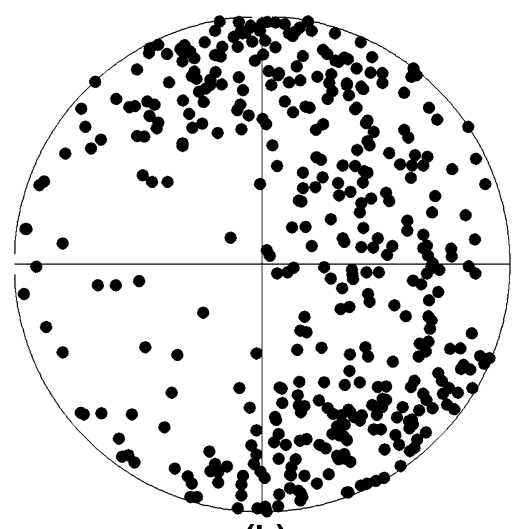

(b)

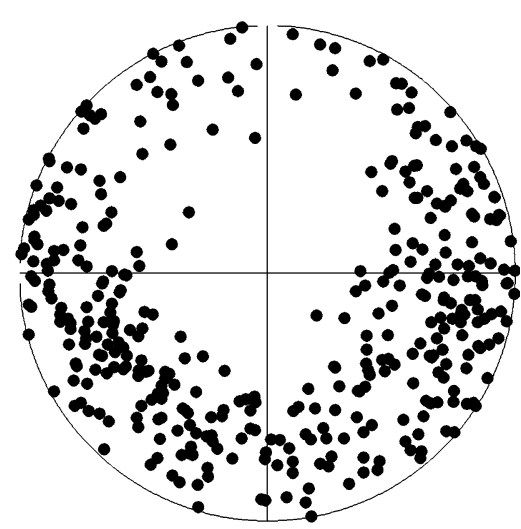

(c)

Fig. 9-Pole figures showing the evolution of the void morphological texture in specimen $A$ as a function of strain: $(a) \varepsilon=0\left(A_{0}\right),(b) \varepsilon=0.09$ $\left(A_{1}\right)$, and $(c) \varepsilon=0.39\left(A_{2}\right)$. 


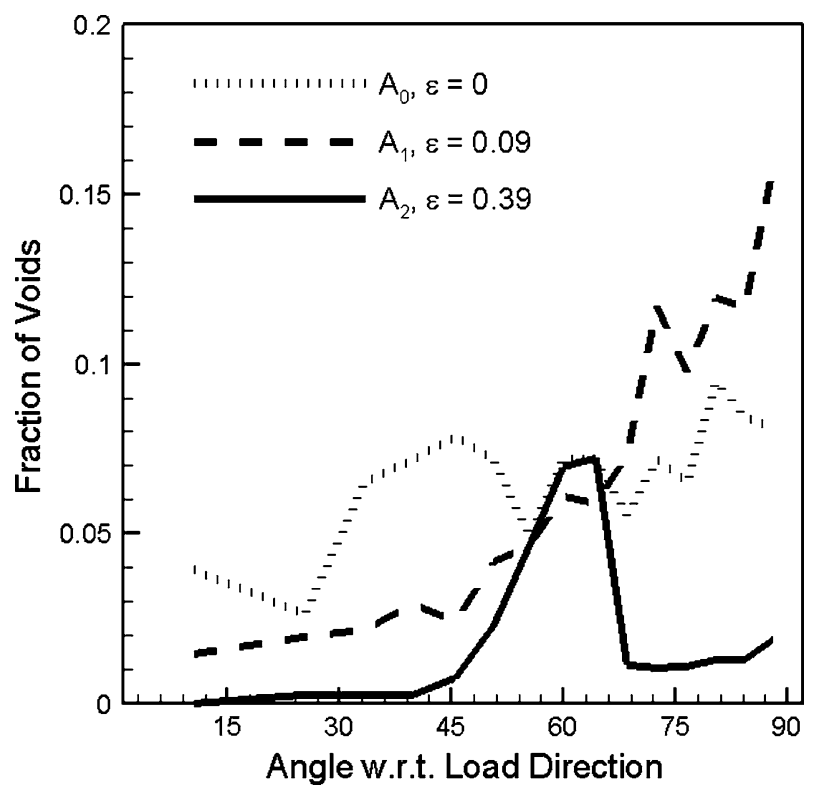

Fig. 10-Effect of strain on orientation of voids relative to loading direction for specimen $A$.

loading direction (i.e., the majority of voids now have their major axis located close to the circumference of the pole plot). This is an indication that at this level of strain, internal damage is increasing via void coalescence and growth across the liquid channels in a direction normal to the loading direction

The degree of void orientation can be quantified by calculating the variation in the major axis angle relative to the loading direction at different levels of strain. Figure 10 presents the results from this calculation for specimen $A$. As can be seen in the figure, voids oriented from 0 to $90 \mathrm{deg}$ are found in the as-cast material, with a mean orientation of $\sim 62 \mathrm{deg}$ and a standard deviation of \pm 19 . With a moderate amount of strain $\left(A_{1}\right)$, the proportion of voids oriented near $90 \mathrm{deg}$ increases dramatically, with the mean orientation increasing to 70 $\operatorname{deg} \pm 17$. Coalescence is clearly occurring at higher strains $\left(A_{2}\right)$, since the mean orientation has increased to 75 deg while the standard deviation has decreased to $\sim 12$ deg. As is shown in Figure 10, there are very few voids at $A_{2}$ with a major axis oriented less than $45 \mathrm{deg}$ to the loading direction. The orientation assessment for both specimen $B\left(55 \mathrm{deg} \pm 20\right.$ for $B_{0}, 68 \mathrm{deg} \pm 18$ for $B_{1}$, and $76 \mathrm{deg} \pm 11$ for $\left.B_{2}\right)$ and specimen $\mathrm{C}\left(58 \mathrm{deg} \pm 22\right.$ for $C_{0}$ and $73 \mathrm{deg} \pm 15$ for $C_{1}$ ) were similar to the results for specimen $A$.

The void spatial orientation plots provide insight into void development in the semisolid. In this material, isolated void growth occurs along directions in which the most strain energy is available for new surface creation, hence the preferred orientation perpendicular to the loading direction. The role of the semisolid material, located preferentially at grain boundaries and at triple points, is probably twofold: (1) as less energy is required to create a surface within the liquid it will act to provide a preferential path for void nucleation and crack propagation; and (2) in certain areas it may also act as a stress riser helping to localize strain. Thus, the residual liquid is able to influence the crack growth and will add some randomness to the crack propagation path. The results shown in Figure 10 provide a clear indication of this, since the mean orientation angle is increased from $A_{0}$ to $A_{1}$, without a corresponding decrease in standard deviation. The tendency of cracks to propagate along paths associated with residual liquid may also contribute to coalescence, and ultimately leads to the low ductility observed at certain critical fractions solid.

\section{Semisolid Failure Mechanism}

The qualitative and quantitative results correlate well with the perceived mechanism by which hot tears form, i.e., tearing along the last to solidify intergranular liquid. For example, the high-resolution image of deformed material shown in Figure 4(c) indicates that damage forms predominantly at the grain boundaries, while the morphological texture analysis shown in Figures 9 and 10 indicates that the damage growth occurs perpendicular to the loading direction. Both of these results are in accord with the unzipping of a thin layer of intergranular liquid. As the liquid phase becomes larger, the unzipping may also occur in the interdendritic region. However, in a well grain-refined material, the intergranular and interdendritic liquid will be connected since the majority of the secondary dendrites terminate at the grain boundaries. As can be seen in Figure 4(c), the length of the void channels joining voids (1), (2), and (3) is similar to the grain size. This similarity in scale provides evidence of tearing along the intergranular liquid as opposed to the interdendritic arms.

The novel experiments presented in this study not only substantiate the prior research showing hot tearing along grain boundary liquid, they also allow for a new semisolid failure mechanism to be proposed. This new mechanism is dependent on the presence or absence of as-cast voids. When an as-cast void is present, it acts as a stress riser and allows strain to be accommodated by growth of this pre-existing damage through the liquid along the grain boundary. In the absence of pre-existing voids, a nucleation step is required. This nucleation step will have to occur as a precursor to hot tearing. Once the stress concentration around the new voids becomes appreciable, they will act identically to as-cast porosity. Furthermore, as can be deduced from Figure 4, it is the combination of a minimal nucleation barrier due to as-cast porosity along with the presence of liquid along the grain boundaries which leads to material with limited ductility, and thus enables growth and coalescence of damage.

The differences in the accumulation of damage observed between samples $A, B$, and $C$ can be explained in terms of their initial as-cast porosity and clearly point to its importance in determining the response of the semisolid material to tensile strain. These results are consistent with the earlier work of Pellini, ${ }^{[3]}$ who showed that hot tears initiate in the thin film of liquid between two solid grains. The new mechanism further suggests that as-cast porosity at the solid-liquid interface will 
enhance hot tearing due to the presence of the free surface, allowing for solid-liquid separation. Farup et $a{ }^{\left[{ }^{[9]}\right.}$ also observed that hot tears formed easily on pores caused by solidification shrinkage. Thus, the current work, which has provided a global quantitative analysis of the effect of voids on hot tearing in an aluminum alloy, verifies that Farup's prior analysis of deformation in a semisolid organic analogue can also be applied to metal systems.

In the absence of a pre-existing porosity, void nucleation is required. The increase in the number of new small voids detected following each of the increments in displacement examined in this study is consistent with nucleation processes occurring. Based on the results shown in Figure 4, it can also be hypothesized that the presence of inclusions, such as oxide films, greatly lowers the barrier for void nucleation both during the casting process as well as during semisolid deformation. The implications of these inclusions as heterogeneous sites for void nucleation and the subsequent decrease in average tensile properties and increase in property variability has previously been discussed. ${ }^{[29-32]}$ However, as shown in the highresolution ESRF tomographic dataset of undeformed as-cast AA5182, the material contains a myriad of small voids and thus it would appear that void nucleation is unnecessary. While the idea of void nucleation is consistent with earlier work by Farup et al. ${ }^{[9]}$ and Fredriksson, ${ }^{[10]}$ it is unclear what effect it has on semisolid ductility.

In summary, the combination of XMT and semisolid deformation is an effective tool for assessing damage evolution in semisolid aluminum alloys. For example, different alloy systems and compositions can be characterized to determine the interplay between internal damage development and final failure. Further, the stress-strain behavior of the material can be measured directly, including both cross-sectional area and internal void growth. The main limitations of the current technique are twofold: (1) the use of interrupted tensile tests on partially remelted material in combination with tomography, rather than continuous in-situ observation of the material as it solidifies; and (2) the inability to conclusively distinguish between void formation by growth of as-cast porosity and nucleation of new voids. These limitations could be addressed by performing the semisolid deformation while simultaneously obtaining a high-resolution tomographic scan using a synchrotron source. These scans would allow for observation of the role of the liquid in void nucleation, growth, and coalescence. However, the combination of specimen heating, tensile deformation, and rotation for tomographic acquisition while remaining outside the X-ray path contains significant technical challenges, which must first be overcome.

\section{CONCLUSIONS}

The first 3-D observations of the development of internal damage with strain in a semisolid commercial Al-Mg alloy have been presented. The combination of interrupted semisolid tensile tests with XMT has enabled new insights to be gained into the processes involved in semisolid material deformation and hot tearing. First, the results of this study strongly support the idea that as-cast voids act as stress risers and allow strain to be accommodated by growth of this preexisting damage through the liquid along grain boundaries. Second, the high-resolution tomography scans and the quantitative assessment of void spatial orientation has shown that voids then grow preferentially in a direction normal to the applied load via void coalescence and unzipping along the liquid at the grain boundaries. Third, the quantitative assessment of void distributions clearly indicates that semisolid deformation and thus hot tearing is greatly affected by the growth of as-cast porosity. At some critical strain, localized void coalescence occurs, leading to a decrease in the void number density and final failure. In the absence of pre-existing voids, it is hypothesized that nucleation of voids would occur to create damage. This "new" damage would then behave identically to the as-cast porosity.

\section{ACKNOWLEDGMENTS}

The authors acknowledge NSERC, EPSRC (GR/ T26344), and Alcan International for financial support. They also thank R. Hamilton and M. Kershaw for assistance with XMT, R. Atwood for assistance in developing the code to calculate each void's eigenvectors, and C. Sinclair for assistance in interpreting the void spatial orientation results. The authors also gratefully acknowledge both Jerome Adrien (MATEIS Laboratory) and the staff members of the ID19 Beam Line ESRF for allowing the high-resolution scan to be performed on the as-cast material.

\section{REFERENCES}

1. J. Campbell: Castings, 2nd ed., Butterworth Heinemann, London, UK, 2003.

2. S. David and T. Debroy: Science, 1992, vol. 257, pp. 497-506.

3. W.S. Pellini: Foundry, 1952, pp. 125-37.

4. U. Feurer: Giesserei-Forschung, 1976, pp. 75-80.

5. D. Warrington and D.G. McCartney: Cast Met., 1991, vol. 3, pp. 202-08.

6. Y.F. Guven and J.D. Hunt: Cast Met., 1988, vol. 1, pp. 104-11.

7. H. Fredriksson and B. Lehtinen: Int. Conf. Solidification and Casting of Metals, Metals Society, Sheffield, United Kingdom, 1977, pp. 260-67.

8. D. Davidson, D. Viano, L. Liming, and D. St. John: in Shape Casting: The John Campbell Symp, M. Tiryakioglu and P.N. Crepeau, eds., TMS, San Francisco, CA, 2005, pp. 175-82.

9. I. Farup, J.M. Drezet, and M. Rappaz: Acta Mater., 2001, vol. 49, pp. 1261-69.

10. H. Fredriksson, M. Haddad-Sabzervar, K. Hansson, and J. Kron: Mater. Sci. Technol., 2005, vol. 21, pp. 521-29.

11. T.S. Piwonka and M.C. Flemings: Trans. TMS-AIME, 1966 , vol. 236, pp. 1157-65.

12. J.P. Hirth, G.M. Pound, and G.R. St. Pierre: Metall. Trans., 1970, vol. 1, pp. 939-45.

13. L.J. Colley, M.A. Wells, and D.M. Maijer: Mater. Sci. Eng. A, 2004, vol. 386 (1-2), pp. 140-48. 
14. W.M. van Haaften, W.H. Kool, and L. Katgerman: Mater. Sci. Eng. A, 2002, vol. 336, pp. 1-6.

15. S. Thompson, S.L. Cockcroft, and M.A. Wells: Mater. Sci. Technol., 2004, vol. 20 (4), pp. 497-504.

16. A.B. Phillion, S.L. Cockcroft, and P.D. Lee: Scripta Mater., 2006, vol. 55 , pp. $489-92$.

17. H. Toda, T. Ohgaki, K. Uesugi, M. Kobayashi, N. Koroda, T. Kobayashi, M. Ninomi, T. Akahori, K. Makii, and Y. Aruga: Metall. Mater. Trans. A, 2006, vol. 37A, pp. 1211-19.

18. E. Maire, J.Y. Buffiere, L. Salvo, J.J. Blandin, W. Ludwig, and J.M. Letang: Adv. Eng. Mater., 2001, vol. 3, pp. 539-46.

19. L. Salvo, P. Cloetens, E. Maire, S. Zabler, J.J. Blandin, J.Y. Buffiere, W. Ludwig, E. Boller, D. Bellet, and C. Josserond: Nucl. Instrum. Meth. Phys. Res., Sect. B, 2003, vol. 200, pp. 273-86.

20. J. Baruchel, J.-Y. Buffiere, P. Cloetens, M.D. Michiel, E. Ferrie, W. Ludwig, E. Maire, and L. Salvo: Scripta Mater., 2006, vol. 55, pp. 41-46.

21. M.D. Abramoff, P.F. Magelhaes, and S.J. Ram: Biophotonics Int., 2004, vol. 11 (7), pp. 36-42.
22. P. Perona and J. Malik: IEEE-PAMI, 1990, vol. 12, pp. 629-39.

23. E. Maire, J.C. Grenier, D. Daniel, A. Baldacci, H. Klocker, and A. Bigot: Scripta Mater., 2006, vol. 55, pp. 123-26.

24. L. Arnberg, L. Backerud, and G. Chai: Solidification Characteristics of Al Alloys-V3 Dendrite Coherency, American Foundry Society, Des Plaines, IL, 1996.

25. P.D. Lee and J.D. Hunt: Acta Mater., 2001, vol. 49, pp. 1383-98.

26. P.D. Lee, R.C. Atwood, R.J. Dashwood, and H. Nagaumi: Mater. Sci. Eng. A, 2002, vol. 328, pp. 213-22.

27. P.D. Lee and J.D. Hunt: Acta Mater., 1997, vol. 45, pp. 4155-69.

28. T.S. Yoo, M.J. Ackerman, W.E. Lorensen, W. Schroeder, V. Chalana, S. Aylward, D. Metaxes, and R. Whitaker: Proc. Medicine Meets Virtual Reality, J. Westwood, ed., Press Amsterdam, 2002, pp. 586-92.

29. N.R. Green and J. Campbell: AFS Trans., 1994, pp. 341-47.

30. J. Mi, R.A. Harding, and J. Campbell: Metall. Mater. Trans. A, 2004, vol. 35A, pp. 2893-902.

31. X. Cao and J. Campbell: Can. Met. Q., 2005, vol. 44, pp. 435-47. 32. J. Campbell: Mater. Sci. Technol., 2006, vol. 22, pp. 127-45. 